NONCOMMUTATIVE GEOMETRY

AND QUANTUM GROUPS

BANACH CENTER PUBLICATIONS, VOLUME 61

INSTITUTE OF MATHEMATICS

POLISH ACADEMY OF SCIENCES

WARSZAWA 2003

\title{
RELATING QUANTUM AND BRAIDED LIE ALGEBRAS
}

\author{
X. GOMEZ* and S. MAJID ${ }^{\dagger}$ \\ School of Mathematical Sciences, Queen Mary, University of London \\ Mile End Rd, London E1 $4 \mathrm{NS}$, UK \\ E-mail: x.gomez@qmul.ac.uk
}

\begin{abstract}
We outline our recent results on bicovariant differential calculi on co-quasitriangular Hopf algebras, in particular that if $\mathfrak{g}_{\Gamma}$ is a quantum tangent space (quantum Lie algebra) for a CQT Hopf algebra $A$, then the space $k \oplus \mathfrak{g}_{\Gamma}$ is a braided Lie algebra in the category of $A$-comodules. An important consequence of this is that the universal enveloping algebra $U\left(\mathfrak{g}_{\Gamma}\right)$ is a bialgebra in the category of $A$-comodules.
\end{abstract}

Introduction. Since the appearance of quantum groups in the mid eighties, and in particular of the pairs of Hopf algebras $\mathcal{O}_{q}(G)$ and $U_{q}(\mathfrak{g})$ associated to semi-simple complex Lie algebras, there has been many attempts to define a corresponding notion of quantum Lie algebra [Wor, Maj-94, LS, DG, Br]. It is remarkable that there is still no fully satisfying answer: all these attempts tend to generalize an aspect of usual Lie algebras, but some other properties that one would expect from the generalization seem to be lost.

The geometric approach to quantum Lie algebras [Wor] tends to reproduce the relation (given by the Lie functor) between a Lie group $G$ and its Lie algebra $\mathfrak{g}=\operatorname{Lie}(G)$, which is defined as the vector space of left (or right) invariant vector fields on $G$. To give a "non-commutative" analogue of this, one has to work out a suitable formalism of differential calculus on a Hopf algebra (the leading example would be $\mathcal{O}_{q}(G)$ ), and this was done in [Wor]. Woronowicz noticed that the (quantum) "tangent space" $\mathfrak{g}_{\Gamma}$ of each such calculus $\Gamma$ comes naturally equipped with maps $\sigma$ (a braiding) and [, ]: $\mathfrak{g}_{\Gamma} \otimes \mathfrak{g}_{\Gamma} \rightarrow \mathfrak{g}_{\Gamma}$ which satisfy identities that generalize the axioms of usual Lie algebras. Thus, these identities can be chosen as the axioms of abstract quantum Lie algebras. In this construction, the classical geometrical picture is preserved, but some familiar features seem to be lost. First a quantum Lie functor, from the category of Hopf algebras to that of bicovariant differential calculi, doesn't seem to exist (given an arbitrary Hopf algebra $A$, there is no canonical

2000 Mathematics Subject Classification: 58B32, 18D10, 20G42, 81R50.

${ }^{*}$ EU Marie Curie Fellow.

$\dagger^{\dagger}$ Royal Society University Research Fellow. 
choice of a bicovariant calculus over it, therefore no canonical choice for "its" quantum Lie algebra). Finally, a quantum Lie algebra has a naturally defined universal enveloping algebra, and it is not obvious that it can be a Hopf algebra in general (as we would expect).

On the other hand, from a purely algebraic point of view, [Maj-94] addressed the problem of finding a Lie-algebra like object generating quantum groups such as $U_{q}(\mathfrak{g})$ along the lines that $U(\mathfrak{g})$ is generated from a Lie algebra $\mathfrak{g}$. The strategy there is first to identify the properties of usual Lie algebras that allow their universal enveloping algebras to be Hopf algebras, then propose a generalization that preserves the Hopf property. As argued in [Maj-94], the properties we are after are better seen if instead of considering a (usual) Lie algebra $\mathfrak{g}$ we consider the space $\tilde{\mathfrak{g}}:=k \gamma \oplus \mathfrak{g}(k$ is the ground field and $\gamma$ is some new vector), of dimension one more. This space can be identify with $U(\mathfrak{g})_{(1)}=k 1+\mathfrak{g}$ in the standard -both algebra and coalgebra- filtration of $U(\mathfrak{g})$, therefore has a natural coalgebra structure $(\Delta, \varepsilon)$, and a "bracket" $\tilde{\mathfrak{g}} \otimes \tilde{\mathfrak{g}} \rightarrow \tilde{\mathfrak{g}}$ obtained from the (in our conventions, left) adjoint action of $U(\mathfrak{g})$ on itself. Generalizing this example and allowing the underlying category to be arbitrary braided category has led to the notion of braided Lie algebra $(\mathcal{L}, \Delta, \varepsilon,[]$,$) , which among other things is already a coalgebra in the category. As shown$ in [Maj-94], a braided Lie algebra $\mathcal{L}$ generates in a canonical way a braided bialgebra $B(\mathcal{L})$ (that is, a bialgebra living in the same braided category as $\mathcal{L}$ ), which is quadratic in the elements of $\mathcal{L}$ and never has an antipode. In case $\mathcal{L}=\tilde{\mathfrak{g}}=k \gamma \oplus \mathfrak{g}$, the braided universal enveloping algebra $B(\tilde{\mathfrak{g}})$ of $\tilde{\mathfrak{g}}$ is related to $U(\mathfrak{g})$ by $U(\mathfrak{g}) \simeq B(\tilde{\mathfrak{g}}) /\langle\gamma-1\rangle$, which happens to have an antipode.

Although they are also meant to generalize Lie algebras, the braided Lie algebras have no geometrical meaning a priori. However, direct observation in [Maj-98] suggests that there should be one, since a braided Lie algebra could be associated to all bicovariant differential calculi over standard quantum groups and finite groups.

In $[\mathrm{GM}]$ we generalized this observation to the class of all co-quasitriangular Hopf algebras and provided some examples. In this note, after recalling briefly some of Woronowicz's constructions [Wor] in section 1, we summarize the main results of [GM] in section 2: given a co-quasitriangular Hopf algebra $A$ and any finite dimensional bicovariant first order differential calculus $\Gamma$ over $A$, the space $\tilde{\mathfrak{g}}_{\Gamma}:=k \oplus \mathfrak{g}_{\Gamma}$ has a natural structure of braided Lie algebra in the category of $A$-comodules (which is braided thanks to coquasitriangularity); moreover, its braided universal enveloping algebra $B\left(\tilde{\mathfrak{g}}_{\Gamma}\right)$ has $U\left(\mathfrak{g}_{\Gamma}\right)$ as bialgebra quotient; finally, $U(\mathfrak{g})$ happens to be quadratic when the calculus is inner (theorem 2.5). We also explain (proposition 2.6) that there is a quantum Lie functor for coquasitriangular Hopf algebras, that is, something that associates to a co-quasitriangular Hopf algebra a preferred bicovariant differential calculus over it (hence also a preferred quantum Lie algebra), and that behaves well with respect to embeddings/projections. However, this functor gives trivial results for the standard deformations of simple matrix groups. The main ingredient of these results is the braided version $\underline{A}$ of $A$ in the category of $A$-comodules. Details of all statements will appear in [GM]. Finally, section 3 gives a (new) generalization of braided Lie algebras of matrix type [Maj-94], following ideas from [Doi] and [GM, Proposition 5.3].

Finally, it is well-known that both quantum and braided Lie algebras have a dimension problem: when one comes to examples, one realizes that there is no quantum or 
braided Lie algebra that could play the role of the "quantization" of $s l(n), s o(n)$ and $s p(n)$, because no candidate has the correct dimension. Because of this there are other approaches to quantum Lie algebras $[\mathrm{LS}],[\mathrm{DG}],[\mathrm{Br}]$, which do tackle this problem but fail to present a clear axiomatic, and only deal with a very small class of Lie algebras (the complex simple ones). It would be very interesting to know how this smaller "generalized" Lie algebras imbed in quantum/braided Lie algebras and to identify what kind of identities/axioms they satisfy inside them, in particular for the series $s o(n)$ and $s p(n)$ (the $\operatorname{sl}(n)$ case is already studied in $[\mathrm{LS}],[\mathrm{GM}]$ ).

\section{Quantum Lie algebras}

Bicovariant FODC and quantum Lie algebras [Wor]. Let $A=(A, \mathrm{~m}, \eta, \Delta, \varepsilon, S)$ be a Hopf algebra over a field $k$, and $(\Gamma, \mathrm{d})$ a bicovariant first order differential calculus (FODC) over it [Wor, KS] $\Gamma$ is a Hopf bimodule over $A$ with left and right coactions written $\Delta_{R}(v)=v^{(0)} \otimes v^{(1)}$ and $\Delta_{L}(v)=v^{(-1)} \otimes v^{(0)}$, and $\mathrm{d}: A \rightarrow \Gamma$ is a derivation, which is also a bicomodule map ( $A$ being seen as the regular bicomodule), and such that the linear map $A \otimes A \rightarrow \Gamma, a \otimes b \mapsto a \mathrm{~d} b$ is surjective. Elements of $A$ can be called functions, those of $\Gamma$ the 1 -forms, and $\mathrm{d}$ the first order differential of the calculus.

There is a canonical projection $\pi_{R}: \Gamma \rightarrow \Gamma, \pi_{R}(v)=v^{(0)} S\left(v^{(1)}\right)$, whose image is exactly the space of right invariant 1-forms (or right cotangent space of $\Gamma$ ) $\Gamma_{R}:=\{v \in$ $\left.\Gamma \mid \Delta_{R}(v)=v \otimes 1\right\} . \Gamma_{R}$ is naturally a left crossed module over $A$ with left action $a \triangleright \omega:=$ $a_{(1)} \omega S\left(a_{(2)}\right),\left(a \in A, \omega \in \Gamma_{R} \subset \Gamma\right)$, and left coaction $\delta_{L}=\left.\Delta_{L}\right|_{\Gamma_{R}}$. By the fundamental theorem of Hopf bimodules, one has $\Gamma \simeq \Gamma_{R} \otimes A$, with tensor product actions and coactions, which are the regular ones for $A$ on both sides, the trivial ones for $\Gamma_{R}$ one the right, and that of $\left(\Gamma_{R}, \triangleright, \delta_{L}\right)$ on the left.

The (right handed) Maurer-Cartan map of the calculus is $\omega_{R}:=\pi_{R} \circ \mathrm{d}: A \rightarrow \Gamma_{R}$, which is also related to the differential by $\mathrm{d}(a)=\omega_{R}\left(a_{(1)}\right) \cdot a_{(2)}, \omega_{R}(a)=\mathrm{d}\left(a_{(1)}\right) S\left(a_{(2)}\right)$ : the data $(\Gamma, d)$ and $\left(\Gamma_{R}, \omega_{R}\right)$ are strictly equivalent. In terms of $\left(\Gamma_{R}, \omega_{R}\right)$, the axioms of a first order differential calculus are that $\left(\Gamma_{R}, \triangleright, \delta_{L}\right)$ is a left crossed module over $A$, and:

$\langle 1\rangle \quad \omega_{R}(a b)=a \triangleright \omega_{R}(b)+\omega_{R}(a) \varepsilon(b)$.

$\langle 2\rangle \quad \delta_{L} \omega_{R}=\left(\mathrm{id} \otimes \omega_{R}\right) \operatorname{ad}_{L}$.

$\langle 3\rangle \quad \omega_{R}: A \rightarrow \Gamma_{R}$ is surjective.

$\left(\operatorname{ad}_{L}: A \rightarrow A \otimes A, \operatorname{ad}_{L}(a)=a_{(1)} S\left(a_{(3)}\right) \otimes a_{(2)}\right.$, is the left adjoint coaction $)$.

Clearly one has from $\langle 1\rangle: \omega_{R}(1)=0$, and therefore by $\langle 3\rangle$ : the restriction of $\omega_{R}$ to $\operatorname{ker} \varepsilon_{A}$ is also surjective. We then see from axioms $\langle 1\rangle$ and $\langle 2\rangle$ that $\omega_{R}$ induces an isomorphism

$$
\left(\Gamma_{R}, \triangleright, \delta_{L}\right) \simeq\left(\operatorname{ker} \varepsilon_{A}, \mathrm{~m}, \operatorname{ad}_{L}\right) / \mathcal{I}
$$

where $\left(\operatorname{ker} \varepsilon_{A}, \mathrm{~m}, \operatorname{ad}_{L}\right)$ is a left crossed module with $a \triangleright b:=\mathrm{m}(a \otimes b)=a b,(a \in A$, $\left.b \in \operatorname{ker} \varepsilon_{A}\right)$, and $\mathcal{I}:=\operatorname{ker} \varepsilon_{A} \cap \operatorname{ker} \omega_{R}$ is a left ideal of $A$ contained in $\operatorname{ker} \varepsilon_{A}$ and a subcomodule of $\left(A, \operatorname{ad}_{L}\right) ; \mathcal{I}$ is uniquely determined by $(\Gamma, \mathrm{d})$ and conversely any such $\mathcal{I}$ defines uniquely a bicovariant FODC over $A$. Thus, $\left(\operatorname{ker} \varepsilon_{A}, \triangleright, \operatorname{ad}_{L}\right)$ is the universal (right) cotangent space for $A$. By definition, the quantum tangent space of the calculus is

$$
\mathfrak{g}_{\Gamma}:=\left\{x \in A^{*} \mid\left\langle 1_{A}, x\right\rangle=0,\langle\mathcal{I}, x\rangle=0\right\},
$$


where $\langle\rangle:, A \otimes A^{*} \rightarrow k$ is the natural pairing. We assume throughout the Hopf dual $A^{\circ} \subset A^{*}$ of $A$ is big enough (if $\langle a, x\rangle=0$ for all $x \in A^{\circ}$, then $a=0$ ), and that $\operatorname{dim}_{k} \mathfrak{g}_{\Gamma}=$ $\operatorname{dim}_{k} \Gamma_{R}$ is finite (it is called the dimension of the calculus). In this case $[\mathrm{KS}], \mathfrak{g}_{\Gamma} \subset A^{\circ}$ and the bilinear form $():, \Gamma \times \mathfrak{g}_{\Gamma} \rightarrow k,\left(\omega_{R}(a) . b, x\right)=\langle a, x\rangle \varepsilon(b)$ identifies $\mathfrak{g}_{\Gamma}$ to $\Gamma_{R}^{*}$ as vector spaces: $\mathfrak{g}_{\Gamma}$ is naturally a right crossed module over $A$. Its crossed module structure $\left(\triangleleft, \delta_{R}\right)$ and the corresponding braiding $\sigma: \mathfrak{g}_{\Gamma} \otimes \mathfrak{g}_{\Gamma} \rightarrow \mathfrak{g}_{\Gamma} \otimes \mathfrak{g}_{\Gamma}\left(\sigma(x \otimes y)=y^{(0)} \otimes x \triangleleft y^{(1)}\right)$ turn out to be:

$$
\begin{aligned}
& x \triangleleft a=\left\langle a, x_{1}\right\rangle x_{2}-\langle a, x\rangle 1_{A^{\circ}}, \quad x^{(0)}\left\langle x^{(1)}, h\right\rangle=[h, x] \\
& \sigma(x \otimes y)=\left[x_{1}, y\right] \otimes x_{2}-[x, y] \otimes 1_{A^{\circ}} .
\end{aligned}
$$

for all $h \in A^{\circ}$. Here, $x_{1} \otimes x_{2}:=\Delta(x), x^{(0)} \otimes x^{(1)}:=\delta_{R}(x)$, and [, ] : $A^{\circ} \otimes A^{\circ} \rightarrow A^{\circ}$ is the linear map defined by $[X, Y]=X_{1} Y S\left(X_{2}\right)$, i.e. $X$ acting on $Y$ by the left adjoint action. Woronowicz has shown that $\left[\mathfrak{g}_{\Gamma}, \mathfrak{g}_{\Gamma}\right] \subset \mathfrak{g}_{\Gamma}$ and that the triple $\left(\mathfrak{g}_{\Gamma},[],, \sigma\right)$ satisfies the following properties:

1. $\sigma$ satisfies the braid relation.

2. Quantum Jacobi identity: $[x,[y, z]]=[[x, y], z]+\sum_{i}\left[y_{i},\left[x_{i}, z\right]\right]$ for all $x, y, z \in \mathfrak{g}_{\Gamma}$, where $\sum_{i} y_{i} \otimes x_{i}=\sigma(x \otimes y)$.

3. Writing $\sigma_{12}=(\sigma \otimes \mathrm{id}), \sigma_{23}=\mathrm{id} \otimes \sigma$, and $C(x \otimes y)=[x, y]$,

$$
\begin{aligned}
& \sigma(\mathrm{id} \otimes C)-(C \otimes \mathrm{id}) \sigma_{23} \sigma_{12}=0, \\
& \sigma(C \otimes \mathrm{id})-(\mathrm{id} \otimes C) \sigma_{12} \sigma_{23}=(C \otimes \mathrm{id})(\mathrm{id} \otimes \sigma)-\sigma(\mathrm{id} \otimes C)(\sigma \otimes \mathrm{id}) .
\end{aligned}
$$

4. Quantum antisymmetry: If $\sum_{i} x_{i} \otimes y_{i} \in \operatorname{ker}(\mathrm{id}-\sigma)$, then $\sum_{i}\left[x_{i}, y_{i}\right]=0$.

These are often taken as the axioms of an abstract (left) quantum Lie algebra $(\mathfrak{g}, \sigma,[]$, $\left(\text { with } \mathfrak{g}_{\Gamma} \text { replaced by arbitrary vector space } \mathfrak{g}\right)^{1}$. Its universal enveloping algebra is then defined as

$$
U(\mathfrak{g}):=T(\mathfrak{g}) /\langle\operatorname{im}(\mathrm{id}-\sigma-[,])\rangle
$$

where $T(\mathfrak{g})$ is the tensor algebra of $\mathfrak{g}$. Axiom 4 ensures that the natural map $\mathfrak{g} \mapsto U(\mathfrak{g})$ is an imbedding and we sometimes identify $\mathfrak{g}$ with its image in $U(\mathfrak{g})$. Clearly the axioms reproduce those of a usual Lie algebra when $\sigma$ is the flip operator $x \otimes y \mapsto y \otimes x$; in this case, axiom 3 becomes a tautology for all $C$ 's and can be forgotten.

Obstructions for a coproduct on $U\left(\mathfrak{g}_{\Gamma}\right)$. It is also well-known that (still in the classical case) $U(\mathfrak{g})$ is a Hopf algebra, in the category of $k$-vector spaces, with coproduct uniquely determined by $\Delta(x)=x \otimes 1+1 \otimes x$, for $x \in \mathfrak{g}$. Moreover, it is such that the algebra filtration induced by the generating space $\mathfrak{g}$ of $U(\mathfrak{g})$ :

$$
U(\mathfrak{g})_{(0)} \subset U(\mathfrak{g})_{(1)} \subset \ldots \subset U(\mathfrak{g})_{(n)} \subset \ldots
$$

with $U(\mathfrak{g})_{(0)}=k 1, U(\mathfrak{g})_{(1)}=k 1+\mathfrak{g}$, etc, is also a coalgebra filtration.

However, this generic coproduct doesn't work for arbitrary quantum Lie algebras. To tackle this, one could think to allow a more general category than just the category of

\footnotetext{
${ }^{1}$ Woronowicz works with left invariant 1-forms and therefore arrives at different identities, corresponding to what we would call a "right quantum Lie algebra". In the following, "quantum Lie algebra" always stands for "left quantum Lie algebra".
} 
vector spaces, but then the only other braiding at hand is $\sigma$ (in Woronowicz's geometrical context this category would be that of right crossed $A$-modules). Assume in the abstract case that $(\mathfrak{g}, \sigma,[]$,$) lives in a k$-linear braided category $(\mathcal{V}, \otimes, \Psi)$ where its braiding is $\Psi_{\mathfrak{g}, \mathfrak{g}}=\sigma$ : we face the problem that the bracket [, ] might fail to be a morphism in the category; if it were, by the naturaliy axiom of the braiding $\Psi$ the identity $\sigma(C \otimes$ id $)-$ (id $\otimes C$ ) $\sigma_{12} \sigma_{23}=0$ should hold, while we only have (1.1). On the other hand, it is easy to check that the condition $\sigma^{2}=1$ is a sufficient condition for the right hand term of (1.1) to vanish. For quantum Lie algebras of differential calculi one can prove:

Proposition 1.1 [GM]. Let $A$ be an arbitrary Hopf algebra, $(\Gamma, \mathrm{d})$ a bicovariant FODC over $A$ and $\left(\mathfrak{g}_{\Gamma}, \sigma,[],\right)$ its quantum Lie algebra. If (and only if) $\sigma^{2}=1$, then $U\left(\mathfrak{g}_{\Gamma}\right)$ is a Hopf algebra in $\mathcal{C}_{A}^{A}$ with coproduct determined uniquely by $\Delta(x)=x \otimes 1+1 \otimes x$ for $x \in \mathfrak{g}_{\Gamma}$.

In the general case, assume that, whatever the category it lives in, $U\left(\mathfrak{g}_{\Gamma}\right)$ has a bialgebra structure such that the algebra filtration (1.2) is also a coalgebra filtration, that is, such that $U\left(\mathfrak{g}_{\Gamma}\right)_{(1)}=k 1+\mathfrak{g}_{\Gamma}$ is a subcoalgebra. Keeping in mind that $\mathfrak{g}_{\Gamma} \simeq\left(\Gamma_{R}\right)^{*} \simeq$ $\left(\operatorname{ker} \varepsilon_{A} / \mathcal{I}\right)^{*}$ as a vector space, it would mean that the space $k \oplus \operatorname{ker} \varepsilon_{A} / \mathcal{I} \simeq A / \mathcal{I}$ has an algebra structure, which we expect to be naturally coming from that of $A$. The required property is that $\mathcal{I}$ is a 2 -sided ideal of $A$, while until now we have only asked $\mathcal{I}$ to be a left ideal. This additional property is no constraint in the commutative case (as when $A$ is some algebra of functions in the usual sense), but becomes a problem in the non commutative one (e.g. quantum groups). We indicate in the next section how this problem can be solved for the class of all co-quasitriangular Hopf algebras.

Remark: extended tangent and cotangent spaces. The spaces $k \oplus \mathfrak{g}_{\Gamma}$ and $k \oplus \operatorname{ker} \varepsilon_{A} / \mathcal{I}$ often appear in the sequel, and it is useful to introduce notations for them. A calculus $(\Gamma, \mathrm{d})$ is called inner if $\mathrm{d}$ is an inner derivation, that is, if there exists $\theta \in \Gamma$ such that for all $a \in A$

$$
\left.\mathrm{d} a=a \theta-\theta a \quad \text { (equivalently: } \quad \omega_{R}(a)=a_{1} \theta S\left(a_{2}\right)-\varepsilon_{A}(a) \theta\right) .
$$

Any bicovariant FODC $(\Gamma, \mathrm{d})$ can be extended to a pair $(\widetilde{\Gamma}, \mathrm{d})$ which satisfies all axioms except $\langle 3\rangle$, with the property that it contains $\Gamma$ as a Hopf sub-bimodule, and that the derivation $A \stackrel{\mathrm{d}}{\longrightarrow} \Gamma \hookrightarrow \widetilde{\Gamma}$ is inner; one takes $\widetilde{\Gamma}=\Gamma \oplus \Theta . A$ as a right $A$-module $(\Theta$ a free variable), with missing structures fixed by: $\Theta$ biinvariant $\left(\Delta_{L}(\Theta)=1 \otimes \Theta\right.$ and $\left.\Delta_{R}(\Theta)=\Theta \otimes 1\right)$, and left action $a \Theta=\mathrm{d} a+\Theta . a$ in $\widetilde{\Gamma} .(\widetilde{\Gamma}, \tilde{d})$ is called the extended bimodule of $\Gamma$ [Wor], and the crossed module $\left(\widetilde{\Gamma}_{R}, \tilde{\nabla}, \tilde{\Delta}_{L}\right)$ of right invariants of $\widetilde{\Gamma}$ is called the (right) extended cotangent space of $\Gamma$. Clearly $\widetilde{\Gamma}=\Gamma_{R} \oplus k \Theta$. Define the map $\tilde{\omega}_{R}: A \rightarrow \widetilde{\Gamma}_{R}$ by $\tilde{\omega}_{R}(a)=a \tilde{\triangleright} \Theta=\omega_{R}(a)+\varepsilon_{A}(a) \Theta$. One has $\operatorname{ker} \tilde{\omega}_{R}=\operatorname{ker} \omega_{R} \cap \operatorname{ker} \varepsilon_{A}=: \mathcal{I}$, and from the axioms $\langle 1-3\rangle$ we see that $\tilde{\omega}_{R}$ induces an isomorphism of left crossed modules

$$
\left(A, \mathrm{~m}, \operatorname{ad}_{L}\right) / \mathcal{I} \stackrel{\simeq}{\longrightarrow} \widetilde{\Gamma}_{R}
$$

With this point of view, the space $k \oplus \mathfrak{g}_{\Gamma}$ is nothing but the extended tangent space of $\Gamma$, which we define as:

$$
\tilde{\mathfrak{g}}_{\Gamma}:=\left\{X \in A^{*}:\langle\mathcal{I}, X\rangle=0\right\}
$$


that is, $\tilde{\mathfrak{g}}_{\Gamma}=k 1_{A^{\circ}} \oplus \mathfrak{g}_{\Gamma}$. The relations between $\Gamma_{R}$ and $\mathfrak{g}_{\Gamma}$ have direct (and simpler) analogues for $\widetilde{\Gamma}_{R}$ and $\tilde{\mathfrak{g}}_{\Gamma}$ : one has $\tilde{\mathfrak{g}}_{\Gamma} \simeq\left(\widetilde{\Gamma}_{R}\right)^{*}$ as right crossed modules over $A$, with right action $\tilde{\triangleleft}$, coaction $\tilde{d}\left(\tilde{d}(X)=: X^{(0)} \otimes X^{(1)}\right)$ and crossed module braiding $\tilde{\sigma}$ below:

$$
X \tilde{\triangleleft} a=\left\langle a, X_{1}\right\rangle X_{2}, \quad X^{(0)}\left\langle X^{(1)}, h\right\rangle=[h, X], \quad \tilde{\sigma}(X \otimes Y)=\left[X_{1}, Y\right] \otimes X_{2},
$$

for all $h \in A^{\circ}$. Many facts about bicovariant FODC take simpler form when expressed in terms of $\widetilde{\Gamma}$. For instance, the axioms 1,2 and 3 of a quantum Lie algebra for $\left(\mathfrak{g}_{\Gamma}, \sigma,[],\right)$ can be advantageously rewritten as follows.

Given a vector space $\mathfrak{g}$, equipped with linear maps $\sigma: \mathfrak{g} \otimes \mathfrak{g} \rightarrow \mathfrak{g} \otimes \mathfrak{g}$ and $[]:, \mathfrak{g} \otimes \mathfrak{g} \rightarrow \mathfrak{g}$, define its extension $(\tilde{\mathfrak{g}}, \tilde{\sigma})$ as follows: set $\tilde{\mathfrak{g}}:=k \gamma \oplus \mathfrak{g}$ and define $\tilde{\sigma}: \tilde{\mathfrak{g}} \otimes \tilde{\mathfrak{g}} \rightarrow \tilde{\mathfrak{g}} \otimes \tilde{\mathfrak{g}}$ by

$$
\begin{aligned}
& \tilde{\sigma}(\gamma \otimes z)=z \otimes \gamma \quad \tilde{\sigma}(z \otimes \gamma)=\gamma \otimes z,(z \in \tilde{\mathfrak{g}}) \\
& \tilde{\sigma}(x \otimes y)=\sigma(x \otimes y)+[x, y] \otimes \gamma \quad(x, y \in \mathfrak{g} \hookrightarrow \tilde{\mathfrak{g}})
\end{aligned}
$$

(When $\mathfrak{g}=\mathfrak{g}_{\Gamma}$ is associated to a bicovariant FODC, the role of $\gamma$ is played by $1_{A^{\circ}}$, and the braiding $\sigma$ on $\mathfrak{g}_{\Gamma}$ and $\tilde{\sigma}$ on $\tilde{\mathfrak{g}}_{\Gamma}$ are indeed related by (1.4).)

Proposition 1.2 [GM]. (i) The following are equivalent:

1. $\tilde{\sigma}$ satisfies the braid relation;

2. the triple $(\mathfrak{g}, \sigma,[]$,$) satisfies axioms 1-3$ of a (left) quantum Lie algebra.

(ii) Let $(\mathfrak{g}, \sigma,[]$,$) be a quantum Lie algebra, (\tilde{\mathfrak{g}}, \tilde{\sigma})$ its extension as above, and $S_{\tilde{\sigma}}(\tilde{\mathfrak{g}}):=$ $T(\tilde{\mathfrak{g}}) /\langle\mathrm{im}(\mathrm{id}-\tilde{\sigma})\rangle$ be the quantum symmetric algebra of $\tilde{\mathfrak{g}}$ with respect to the braid operator $\tilde{\sigma}$. The algebras $U(\mathfrak{g})$ and $S_{\tilde{\sigma}}(\tilde{\mathfrak{g}})$ are related by

$$
U(\mathfrak{g}) \simeq S_{\tilde{\sigma}}(\tilde{\mathfrak{g}}) /\langle\gamma-1\rangle
$$

(iii) Assume moreover that there exists a subspace $\mathcal{L}$ of $\tilde{\mathfrak{g}}$ such that $\tilde{\mathfrak{g}}=k \gamma \oplus \mathcal{L}$ and $\tilde{\sigma}(\mathcal{L} \otimes \mathcal{L}) \subset \mathcal{L} \otimes \mathcal{L}$. Then $S_{\tilde{\sigma}}(\tilde{\mathfrak{g}}) \simeq k[\gamma] \otimes S_{\tilde{\sigma}_{\mid \mathcal{L}}}(\mathcal{L})$, and

$$
U(\mathfrak{g}) \simeq S_{\tilde{\sigma}_{\mid \mathcal{L}}}(\mathcal{L}) \simeq S_{\sigma}(\mathfrak{g})
$$

is itself a quantum symmetric algebra.

Observe that $\gamma$ is central in $S_{\tilde{\sigma}}(\tilde{\mathfrak{g}})$. Therefore, by (1.5), if we can find a bialgebra structure on $S_{\tilde{\sigma}}(\tilde{\mathfrak{g}})$ (in whatever category) for which $\gamma$ is grouplike, then we immediately get one on $U(\mathfrak{g})$ as well. Note that in $(i i i)$ the spaces $\mathcal{L}$ and $\mathfrak{g}$ must have the same dimension. This pathology doesn't happen in the case of usual Lie algebras (except for abelian ones) and one can ask when this happens in the context of differential calculi. One has the following criterion.

TheOREM $1.3[\mathrm{GM}]$. Let $(\Gamma, \mathrm{d})$ be a finite dimensional bicovariant FODC over some Hopf algebra $A$. If $\mathrm{d}$ is inner and implemented by a biinvariant element $\theta$, then the subspace

$$
\mathcal{L}:=\left\{x \in \tilde{\mathfrak{g}}_{\Gamma} \mid\langle 1-\hat{\theta}, x\rangle=0\right\}
$$

where $\hat{\theta} \in \operatorname{ker} \varepsilon_{A}$ is any preimage of $\theta$ under $\omega_{R}$, satisfies the properties of (iii) in proposition 1.2 and one has an algebra isomorphism

$$
U\left(\mathfrak{g}_{\Gamma}\right) \simeq S_{\tilde{\sigma}}(\mathcal{L})
$$


2. The co-quasitriangular case. Let $(A, \mathbf{r})$ be a co-quasitriangular Hopf algebra, that is, the linear map $\mathbf{r}: A \otimes A \rightarrow k$ is convolution invertible, intertwines the multiplication of $A$ and its opposite $\left(\mathrm{m}^{o p} * \mathbf{r}=\mathbf{r} * \mathrm{~m}, *\right.$ being the convolution product), and satisfies $\mathbf{r}(a, b c)=\mathbf{r}\left(a_{2}, b\right) \mathbf{r}\left(a_{1}, c\right)$, and $\mathbf{r}(a b, c)=\mathbf{r}\left(a, c_{1}\right) \mathbf{r}\left(b, c_{2}\right)$ for all $a, b, c \in A$ (with notation $a_{1} \otimes a_{2}=\Delta(a)$, etc). The convolution inverse of $\mathbf{r}$ is then $\overline{\mathbf{r}}(a, b):=\mathbf{r}(S(a), b)$. It is convenient to introduce the linear maps $\mathbf{r}_{1}, \mathbf{r}_{2}: A \rightarrow A^{\circ}$ by $\left\langle b, \mathbf{r}_{1}(a)\right\rangle=\mathbf{r}(a, b)$ and $\left\langle b, \mathbf{r}_{2}(a)\right\rangle=\mathbf{r}(b, a)$. Then $\mathbf{r}_{1}$ is an algebra/anticoalgebra map, while $\mathbf{r}_{2}$ is an antialgebra/coalgebra map.

Recall that thanks to $\mathbf{r}$, the category ${ }^{A} \mathcal{M}$ is braided, with braiding given by $(v \otimes w) \mapsto$ $\mathbf{r}\left(w^{(-1)}, v^{(-1)}\right) w^{(0)} \otimes v^{(0)}$, and it imbeds in the category ${ }_{A}^{A} \mathcal{C}$ via the monoidal functor $\mathcal{F}_{\mathbf{r}}$ which sends a left comodule $\left(M, \delta_{L}\right)$ to the left crossed module $\left(M, \boldsymbol{}_{\mathbf{r}}, \delta_{L}\right)$, where $a>_{\mathbf{r}} m:=\left\langle m^{(-1)}, \mathbf{r}_{2}(a)\right\rangle m^{(0)}$.

The (Hopf) algebra $\underline{A}$. The Hopf algebra $A$ has a braided version $\underline{A}$ in ${ }^{A} \mathcal{M}$ defined as follows. First $\underline{A}=A$ (as a vector space) is regarded as an object in ${ }^{A} \mathcal{M}$ by the left adjoint coaction $\operatorname{ad}_{L}$ (in the following, $a^{(-1)} \otimes a^{(0)}$ always stands for $\operatorname{ad}_{L}(a), a \in A$ ). Define the linear maps $\underline{\mathrm{m}}: A \otimes A \rightarrow A, \underline{\eta}=\eta: k \rightarrow A, \underline{S}: A \rightarrow A$ by

$$
\begin{aligned}
\underline{\mathrm{m}}(a \otimes b)=a_{\underline{.}} b & :=a_{1}\left(S\left(a_{2}\right) \boldsymbol{\vee}_{\mathbf{r}} b\right)=a_{1} b_{2}\left\langle b_{1} S\left(b_{3}\right), \mathbf{r}_{2} S\left(a_{2}\right)\right\rangle \\
\underline{S}(a) & :=a_{1} \triangleright_{\mathbf{r}} S\left(a_{2}\right) .
\end{aligned}
$$

$\underline{A}:=(A, \underline{\mathrm{m}}, \underline{\eta}, \Delta, \varepsilon, \underline{S})$ is a Hopf algebra in ${ }^{A} \mathcal{M}[\mathrm{Maj}-93, \mathrm{Maj}]$. Recall that this means essentially the same as a usual Hopf algebra, provided we give $\underline{A} \otimes \underline{A}$ the appropriate algebra and coalgebra structures [Maj-LN]: one has to take into account the categorical braiding on $\underline{A}$ in ${ }^{A} \mathcal{M}$ given by $\Psi_{\mathbf{r}}(a \otimes b)=\mathbf{r}\left(b^{(-1)}, a^{(-1)}\right) b^{(0)} \otimes a^{(0)}$; also very important, the structure maps of $\underline{A}$ (as for any Hopf algebra in a braided category) are morphisms in this category, here ${ }^{A \mathcal{M}}$. This means that $\underline{\mathrm{m}}, \underline{\varepsilon}$ and $\underline{S}$ are all intertwiners of the adjoint comodule (in the appropriate sense for each map). Our first observation is the following:

Proposition $2.1[\mathrm{GM}]$. The property " $\mathrm{m}$ op $=\mathbf{r} * \mathrm{~m} * \overline{\mathbf{r}}$ " of the multiplication of $A$ implies the $\Xi$-commutativity "m $\circ \Xi=\underline{\mathrm{m}}$ ” for $\underline{A}$, where $\Xi: A \otimes A \rightarrow A \otimes A$,

$$
\Xi(a \otimes b):=b_{2} \otimes a^{(0)}\left\langle a^{(-1)}, \mathbf{r}_{1}\left(b_{1}\right) \mathbf{r}_{2}\left(b_{3}\right)\right\rangle,
$$

is invertible and satisfies the braid relation. Moreover, for a subcomodule $\mathcal{I}$ of $\left(A, \operatorname{ad}_{L}\right)$, the following are equivalent:

$$
\text { (a) } A \mathcal{I} \subset \mathcal{I}, \quad \text { (b) } A . \mathcal{I} \subset \mathcal{I}, \quad \text { (c) } \mathcal{I} . A \subset \mathcal{I}
$$

From this we see that that when $A$ is co-quasitriangular, an extended cotangent space $\widetilde{\Gamma} \simeq A / \mathcal{I}$ has a natural algebra structure (that of $\underline{A} / \mathcal{I}$ ), and therefore the dual $\tilde{\mathfrak{g}}_{\Gamma}$ has a natural coalgebra structure.

$\tilde{\mathfrak{g}}_{\Gamma}$ is a braided Lie algebra. It remains to show that this coalgebra structure on $\tilde{\mathfrak{g}}_{\Gamma}=$ $k \oplus \mathfrak{g}_{\Gamma}$ does induce a bialgebra structure on $U\left(\mathfrak{g}_{\Gamma}\right)$, in a category to be made precise. We do this by showing that this coalgebra structure on $\tilde{\mathfrak{g}}_{\Gamma}$, together with the "bracket" given by the left adjoint action, do fulfill the axioms of a braided Lie algebra [Maj-94] in the category of right $\mathcal{A}$-comodules; we then apply the general results of [Maj-94]. 
Definition 2.2. Let $(\mathcal{V}, \otimes, \Psi)$ be a braided tensor category. A (left) braided Lie coalgebra in $\mathcal{V}$ is an algebra $(\mathcal{A}, \mu, \eta)$ in the category endowed with a morphism (the braided Lie cobracket) $\delta: \mathcal{A} \rightarrow \mathcal{A} \otimes \mathcal{A}$ satisfying the axioms below:

(C1)

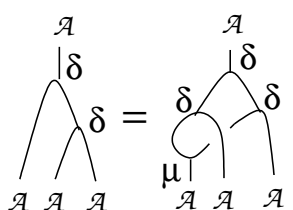

(C2)

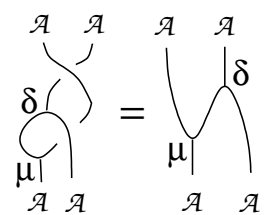

(C3)

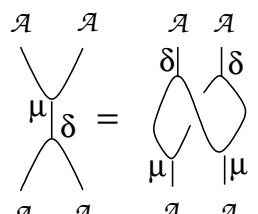

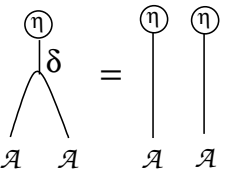

Then we have the following result:

Proposition $2.3[\mathrm{GM}]$. The algebra $(\underline{A}, \underline{\mathrm{m}}, \underline{\eta})$, endowed with the cobracket $\delta=\operatorname{ad}_{L}$ : $\underline{A} \rightarrow \underline{A} \otimes \underline{A}$ is a braided Lie coalgebra in the category ${ }^{A} \mathcal{M}$ of left A-comodules.

If $\mathcal{I}$ is a crossed submodule of $\left(A, \mathrm{~m}, \mathrm{ad}_{L}\right)$, then $\underline{A} / \mathcal{I}$ is also a braided Lie coalgebra in ${ }^{A} \mathcal{M}$ (with structure maps induced from that of $\left(\underline{A}, \underline{\mathrm{m}}, \eta, \delta=\operatorname{ad}_{L}\right)$ ).

Hence, if $(\Gamma, \mathrm{d})$ is a bicovariant FODC over $(A, \mathbf{r})$, then its extended cotangent space $\widetilde{\Gamma}_{R}$ has a natural structure of braided Lie coalgebra in ${ }^{A} \mathcal{M}$ (that of $\underline{A} / \mathcal{I}$ ).

If $\underline{A} / \mathcal{I}$ is finite dimensional (which we assume), we can dualize the properties of $\underline{A} / \mathcal{I}$ to properties of its dual vector space $\tilde{\mathfrak{g}}_{\Gamma} \simeq(\underline{A} / \mathcal{I})^{*}$. Following our point of view to see $\widetilde{\Gamma}_{R}$ as a left $A$-comodule and $\tilde{\mathfrak{g}}_{\Gamma}$ as a right one (rather than both left by using the antipode), the correct conventions are as follows. Extending the pairing between $A$ and $\tilde{\mathfrak{g}}_{\Gamma}$ to a pairing between $A \otimes A$ and $\tilde{\mathfrak{g}}_{\Gamma} \otimes \tilde{\mathfrak{g}}_{\Gamma}$ in the usual way $(\langle a \otimes b, x \otimes y\rangle:=\langle a, x\rangle\langle b, y\rangle)$, we obtain uniquely determined maps $\underline{\Delta}: \tilde{\mathfrak{g}}_{\Gamma} \rightarrow \tilde{\mathfrak{g}}_{\Gamma} \otimes \tilde{\mathfrak{g}}_{\Gamma}, \underline{\varepsilon}: \tilde{\mathfrak{g}}_{\Gamma} \rightarrow k$ and $[]:, \tilde{\mathfrak{g}}_{\Gamma} \otimes \tilde{\mathfrak{g}}_{\Gamma} \rightarrow \tilde{\mathfrak{g}}_{\Gamma}$ defined for all $X, Y \in \tilde{\mathfrak{g}}_{\Gamma}$ by:

$\forall a, b \in A, \quad\langle a \otimes b, \underline{\Delta}(X)\rangle=\langle a \underline{.} b, X\rangle, \underline{\varepsilon}(X)=\left\langle\underline{\eta}\left(1_{A}\right), X\right\rangle, \quad\langle a,[X, Y]\rangle=\left\langle\operatorname{ad}_{L}(a), X \otimes Y\right\rangle$, and the braiding $\Psi_{\mathbf{r}}$ on $\tilde{\mathfrak{g}}_{\Gamma}$ in $\mathcal{M}^{A}$ is given by

$$
\begin{aligned}
\forall a, b \in A, \quad\left\langle a \otimes b, \Psi_{\mathbf{r}}(X \otimes Y)\right\rangle & =\left\langle\Psi_{\mathbf{r}}(a \otimes b), X \otimes Y\right\rangle \\
& =\mathbf{r}\left(b^{(-1)}, a^{(-1)}\right)\left\langle b^{(0)} \otimes a^{(0)}, X \otimes Y\right\rangle .
\end{aligned}
$$

REMARK. The coproduct $\underline{\Delta}$ and braiding $\Psi_{\mathbf{r}}$ on $\tilde{\mathfrak{g}}_{\Gamma}$ cannot be written explicitly in general. However, when $\mathbf{r}=\mathcal{R} \in A^{\circ} \otimes A^{\circ}$, one has:

$$
\triangleq(X)=X_{1} S\left(\mathcal{R}^{(2)}\right) \otimes\left[\mathcal{R}^{(1)}, X_{2}\right], \quad \Psi_{\mathbf{r}}(X \otimes Y)=\left[\mathcal{R}^{(2)}, Y\right] \otimes\left[\mathcal{R}^{(1)}, X\right]
$$

where $\mathcal{R}^{(1)} \otimes \mathcal{R}^{(2)}=\mathcal{R}$ in usual notations.

The correct statement is that $\left(\tilde{\mathfrak{g}}_{\Gamma}, \underline{\Delta}, \underline{\varepsilon},[],\right)$ is a left braided Lie algebra in the category of right $A$-comodules, in the following sense:

Definition 2.4 [Maj-94]. A (left) braided Lie algebra in a braided category $(\mathcal{V}, \otimes, \Psi)$ is a coalgebra $(L, \Delta, \varepsilon)$ in the category, equipped with a morphism in $\mathcal{V}$ (the 


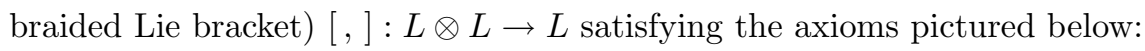

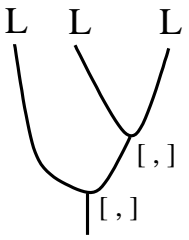

$\mathrm{L}$

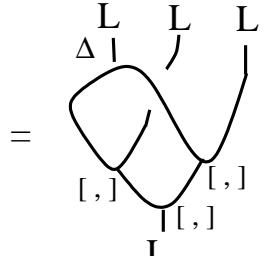

$\mathrm{L}$
(L2)

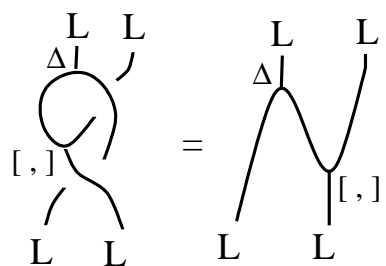

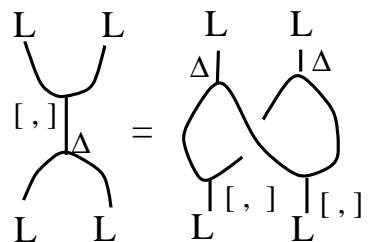

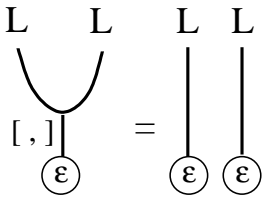

Axiom (L1) is called the left braided Jacobi identity, (L2) weak braided cocommutativity,

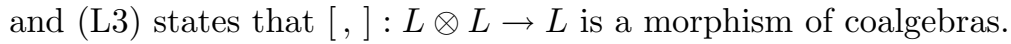

A braided Lie subalgebra of $L$ is a subcoalgebra $M$ such that $[M, M] \subset M$. A morphism of braided Lie algebras in $\mathcal{V}$ is a morphism of coalgebras $\phi: L_{1} \rightarrow L_{2}$ such that $[,]_{2} \circ$ $(\phi \otimes \phi)=\phi \circ[,]_{1}$.

Some general properties of braided Lie algebras. Let $(L, \Delta, \varepsilon,[]$,$) be an arbitrary$ braided Lie algebra in a braided category $(\mathcal{V}, \otimes, \Psi)$. The morphism $\Upsilon: L \otimes L \rightarrow L \otimes L$ given by $\Upsilon \doteq([,] \otimes \mathrm{id}) \circ(\mathrm{id} \otimes \Psi) \circ(\Delta \otimes \mathrm{id})$, or in diagrammatic form $(Y$ in the box means $\Upsilon$ ):

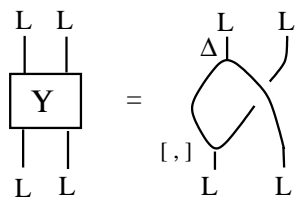

is called the canonical braiding of $(L, \Delta, \varepsilon,[]$,$) . It has the following properties [Maj-94,$ Maj-95, GM]:

1. $\Upsilon: L \otimes L \rightarrow L \otimes L$ is a morphism of coalgebras (in the category).

2. $\Upsilon$ satisfies the braid relation.

3. The braided universal enveloping algebra of $L, B(L):=S_{\Upsilon}(L)=T(L) /\langle\operatorname{im}(\mathrm{id}-\Upsilon)\rangle$, is a (quadratic) bialgebra in $\mathcal{V}$, without antipode.

Note that these properties can be deduced from the axioms of an abstract braided Lie algebra; when $L=\tilde{\mathfrak{g}}_{\Gamma}$ is the extended tangent space of a bicovariant FODC $\Gamma$ over some $(A, \mathbf{r})$, the second property of $\Upsilon$ is in fact obvious, since one finds that

$$
\Upsilon(X \otimes Y)=\left[X_{1}, Y\right] \otimes X_{2}
$$

for all $X, Y \in \tilde{\mathfrak{g}}_{G}$, where $X_{1} \otimes X_{2}=\Delta(X)$ is the (usual) coproduct of $X \in \tilde{\mathfrak{g}}_{\Gamma} \subset A^{\circ}$ : in this case, $\Upsilon$ is in fact identical to the braiding $\tilde{\sigma}$ of the crossed module $\tilde{\mathfrak{g}}_{\Gamma}(1.3)$. Let us stress that the extended quantum tangent space $\tilde{\mathfrak{g}}_{\Gamma}$ is now equipped with two braidings: 
$\Psi_{\mathbf{r}}$, the braiding in the category $\mathcal{M}^{A}$ it lives in as a braided Lie algebra $(2.1)$, and $\Upsilon=\tilde{\sigma}$, the braiding in $\mathcal{C}_{A}^{A}(2.2)$.

Relation between $B\left(\tilde{\mathfrak{g}}_{\Gamma}\right)$ and $U\left(\mathfrak{g}_{\Gamma}\right)$. The braided universal enveloping algebra of the braided Lie algebra $\tilde{\mathfrak{g}}_{\Gamma}$ is the quantum symmetric algebra of $\tilde{\mathfrak{g}}_{\Gamma}$ with respect to the braid operator $\Upsilon=\tilde{\sigma}$. In view of (1.5) and the remark following proposition 1.2 we obtain the first part of

TheOREm $2.5[\mathrm{GM}]$. Let $(A, \mathbf{r})$ be a co-quasitriangular Hopf algebra and $(\Gamma, \mathrm{d})$ be a finite dimensional bicovariant FODC over $A$.

(i) The extended tangent space $\tilde{\mathfrak{g}}_{\Gamma}$ is a braided Lie algebra in $\mathcal{M}^{A}$, and $U\left(\mathfrak{g}_{\Gamma}\right) \simeq$ $B\left(\tilde{\mathfrak{g}}_{\Gamma}\right) /\left\langle 1_{A^{\circ}}-1\right\rangle$ is a bialgebra in $\mathcal{M}^{A}$.

(ii) If $\mathrm{d}$ is inner and implemented by a biinvariant element $\theta$, then the subspace $\mathcal{L}$ of $\tilde{\mathfrak{g}}_{\Gamma}$ defined in (1.6) is a braided Lie subalgebra of $\tilde{\mathfrak{g}}_{\Gamma}$, and one has a bialgebra isomorphism

$$
U\left(\mathfrak{g}_{\Gamma}\right) \simeq S_{\tilde{\sigma}_{\mid \mathcal{L}}}(\mathcal{L})
$$

REMARKs. (i) Let us stress again that:

- The axioms of a quantum Lie algebra $(\mathfrak{g}, \sigma,[]$,$) generalize those of a usual Lie$ algebra.

- Those of a braided Lie algebras are based on the model $\tilde{\mathfrak{g}}=k \oplus \mathfrak{g}$, where $\mathfrak{g}$ is a usual Lie algebra.

But it turns out that important examples of braided Lie algebras do not look at all like this model: for instance the braided Lie subalgebras $\mathcal{L}$ that appear under hypothesis $(i i)$ of the theorem can be very different from the model (e.g. they might have no grouplike element). See for instance the matrix braided Lie algebras $\mathcal{L}(R)$ associated to numerical solutions of the Yang-Baxter equation [Maj-94] or their generalization in the next section.

The two notions are therefore not equivalent: if $(\mathfrak{g}, \sigma,[]$,$) is an abstract quantum Lie$ algebra, the space $k \oplus \mathfrak{g}$ need not have a structure of a braided Lie algebra, and conversely if $(\mathcal{L}, \Delta, \varepsilon,[]$,$) is a braided Lie algebra, \operatorname{ker} \varepsilon \subset \mathcal{L}$ is not necessarily a quantum Lie algebra in Woronowicz's sense (see sections 1 and 2 of [GM] for more details).

(ii) Observe that (from the classifications [Maj-98, BS, KS]), the point (ii) of the theorem concerns all bicovariant FODC over the algebra of functions on a finite group, or over quantized function algebras of $S L(n)$ and $S p(n)$ (and probably of all other classical matrix groups).

A quantum Lie functor for co-quasitriangular Hopf algebras. The previous discussion suggests an analogue of the Lie functor for Lie groups. If $G$ is some algebraic group, the Hopf algebra $A=\mathcal{O}(G)$ of polynomial functions on $G$ has a distinguished bicovariant FODC, for which $\mathcal{I}_{\Gamma}:=\left(\operatorname{ker} \varepsilon_{A}\right)^{2}$ : it is clearly a 2 -sided ideal of $A$ contained in $\operatorname{ker} \varepsilon_{A}$, and the property $\operatorname{ad}_{L}\left(\operatorname{ker} \varepsilon_{A}\right)^{2} \subset A \otimes \operatorname{ad}_{L}\left(\operatorname{ker} \varepsilon_{A}\right)^{2}$ follows from the analogous one for $\operatorname{ker} \varepsilon_{A}$ and the commutativity of $A$. The associated tangent space is $\mathfrak{g}_{\Gamma}=\left\{x \in \operatorname{ker} \varepsilon_{A^{\circ}} \mid\left\langle\left(\operatorname{ker} \varepsilon_{A}\right)^{2}, x\right\rangle=0\right\}=\operatorname{Prim}\left(A^{\circ}\right)$, the space of primitive elements of $A^{\circ}$, i.e. the Lie algebra of $G$.

In the non-commutative case, $\left(\operatorname{ker} \varepsilon_{A}\right)^{2}$ is no longer closed under $\operatorname{ad}_{L}$, but things work well if we replace the product of $A$ by that of $\underline{A}$ (recall that all structure maps of $\underline{A}$, 
including its multiplication, intertwine the adjoint coaction $\left.\operatorname{ad}_{L}\right)$. However, the product of $\underline{A}$ depends on the choice of the co-quasitriangular structure, therefore the pair $(A, \mathbf{r})$ should be considered rather than $A$ alone.

Let $\mathcal{D}$ be the category of bicovariant first order differential calculi: its objects are triples $(A, \Gamma, \mathrm{d})$ where $A$ is a Hopf algebra and $(\Gamma, \mathrm{d})$ a bicovariant first order differential calculus over $A$. Morphisms are pairs $\left(\varphi^{0}, \varphi^{1}\right):\left(A, \Gamma_{A}, \mathrm{~d}_{A}\right) \rightarrow\left(B, \Gamma_{B}, \mathrm{~d}_{B}\right)$ such that $\varphi^{0}: A \rightarrow B$ is a Hopf algebra homomorphism and $\varphi^{1}:\left(\Gamma_{A}, \mathrm{~d}_{A}\right) \rightarrow\left(\Gamma_{B}, \mathrm{~d}_{B}\right)$ is a morphism of Hopf bimodules (over $A$ ) such that $\varphi^{1} \circ \mathrm{d}_{A}=\mathrm{d}_{B} \circ \varphi^{0}$; equivalently, such that $\varphi^{1} \circ \omega_{R, A}=\omega_{R, B} \circ \varphi^{0}$. Because of the surjectivity axiom $\langle 3\rangle$ of a bicovariant FODC, $\varphi^{1}$, if it exists, is uniquely determined by $\varphi^{0}$. The condition of existence is easily seen to be that $\varphi^{0}\left(\mathcal{I}_{\Gamma_{A}}\right) \subset \mathcal{I}_{\Gamma_{B}}$. Let $\mathcal{C} \mathcal{Q} \mathcal{T}$ be the category of co-quasitriangular Hopf algebras: it consists of pairs $(A, \mathbf{r})$ where $A$ is a Hopf algebra and $\mathbf{r}$ is a co-quasitriangular structure on $A$. Morphisms are Hopf algebra morphisms $\varphi: A \rightarrow B$ satisfying $\mathbf{r}_{B} \circ(\varphi \otimes \varphi)=\mathbf{r}_{A}$.

Proposition $2.6[\mathrm{GM}]$. There is an exact functor $\mathbf{L 1}: \mathcal{C Q T} \rightarrow \mathcal{D}$, which sends $(A, \mathbf{r})$ to $(A, \Gamma(\mathbf{r}), \mathrm{d})$ where $(\Gamma(\mathbf{r}), \mathrm{d})$ is the bicovariant $F O D C$ over $A$ whose associated left ideal is $\mathcal{I}(\mathbf{r}):=\operatorname{ker} \varepsilon_{A}$. $\operatorname{ker} \varepsilon_{A}$ (the product in $\underline{A}=\underline{A}(\mathbf{r})$ ).

For algebras of functions on finite groups (with $\mathbf{r}=\varepsilon_{A} \otimes \varepsilon_{A}$ ), it gives zero, which is the correct answer. However it also gives zero for $\mathcal{O}_{q}(S L(n))$ for instance ( $q$ not a root of unity), and in general behaves badly for factorizable Hopf algebras. However, it recovers the (known) non-trivial results for softer (triangular) deformations of $S L(n)$ [GM].

3. A generalization of matrix type braided Lie algebras. We give without proof a generalization of matrix type braided Lie algebras of [Maj-94, §5], in the same spirit as Doi generalized FRT bialgebras associated to numerical solutions of the quantum Yang-Baxter equation [Doi], and directly inspired from [Maj-94, §5] and [GM, proposition 5.3]. The explicit proof is very lengthy but straightforward.

Let $(C, \Delta, \varepsilon)$ be a coalgebra over a field $k$ and $r: C \otimes C \rightarrow k$ a "Yang-Baxter form" on $C$, that is, a linear map satisfying the identity

$$
r_{12} * r_{13} * r_{23}=r_{23} * r_{13} * r_{12}
$$

for all $a, b, c \in C$, where $r_{12}=r \otimes \varepsilon, r_{23}=\varepsilon \otimes r$ and $r_{13}(a \otimes b \otimes c):=r(a \otimes c) \varepsilon(b)$. We assume that $r$ is biinvertible in the sense that its convolution inverse $\bar{r}$ exists, as well as a (uniquely determined) map $\tilde{r}: C \otimes C \rightarrow k$ such that $r\left(a_{1}, b_{2}\right) \tilde{r}\left(a_{2}, b_{1}\right)=\tilde{r}\left(a_{1}, b_{2}\right) r\left(a_{2}, b_{1}\right)=\varepsilon(a) \varepsilon(b)$ for all $a, b \in A$.

Let us define a braided Lie algebra $(C, \Delta, \varepsilon,[],, \Psi)$ over a field $k$ as a coalgebra

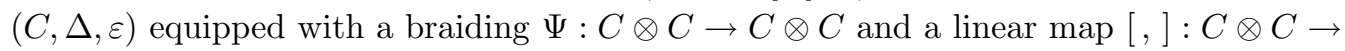
$C$ satisfying axioms (L1)(L2)(L3) of definition 2.4 (with $\Psi$ in place of the categorical braiding), plus all axioms corresponding to the naturality of the braiding with respect to the maps $\Delta, \varepsilon$ and [, ]: e.g. $(\Delta \otimes$ id $) \circ \Psi=\Psi_{12} \Psi_{23} \circ(\Delta \otimes$ id), see exhaustive list in [Maj-94, Fig. 12].

Proposition 3.1. Let $(C, \Delta, \varepsilon)$ be a coalgebra over $k$ equipped with two biinvertible Yang-Baxter forms $r$ and $s$. Then $\left(C, \Delta, \varepsilon,[,]_{r, s}, \Psi_{r}\right)$ is a braided Lie algebra with "cat- 
egorical braiding" $\Psi_{r}$, canonical braiding $\Upsilon_{r, s}$ and braided Lie bracket $[,]_{r, s}$ given by

$$
\begin{aligned}
\Psi_{r}(a \otimes b) & =b_{3} \otimes a_{3} r\left(a_{1}, b_{2}\right) \bar{r}\left(a_{2}, b_{4}\right) \tilde{r}\left(a_{5}, b_{1}\right) r\left(a_{4}, b_{5}\right), \\
\Upsilon_{r, s}(a \otimes b) & =b_{3} \otimes a_{3} \bar{s}_{21}\left(a_{1}, b_{2}\right) s_{21}\left(a_{2}, b_{4}\right) \tilde{r}\left(a_{5}, b_{1}\right) r\left(a_{4}, b_{5}\right), \\
{[a, b]_{r, s} } & =(\mathrm{id} \otimes \varepsilon) \Upsilon_{r, s}(a \otimes b) \\
& =b_{3} \bar{s}_{21}\left(a_{1}, b_{2}\right) s_{21}\left(a_{2}, b_{4}\right) \tilde{r}\left(a_{4}, b_{1}\right) r\left(a_{3}, b_{5}\right) .
\end{aligned}
$$

REMARKs. The matrix type braided Lie algebras $\mathcal{L}(R)$ of [Maj-94] correspond to the case where $C$ is a matrix coalgebra. As shown in [GM], the braided Lie algebras arising from bicovariant FODC over co-quasitriangular Hopf algebras by the construction of [KS, Chap. 14] are all homomorphic images of some $\left(C, \Delta, \varepsilon,[,]_{r, s}, \Psi_{r}\right)$ as above.

\section{References}

[BS] P. Bauman and F. Schmidt, Classification of bicovariant differential calculi over quantum groups (a representation-theoretic approach), Comm. Math. Phys. 194 (1998), $71-86$.

[Br] M. Bremner, Quantum deformations of simple Lie algebras, Canad. Math. Bull. 40 (1996), 143-148.

[DG] G. W. Delius and M. D. Gould, Quantum Lie algebras, their existence, uniqueness and q-antisymmetry, Comm. Math. Phys. 185 (1997), 709-722.

[Doi] Y. Doi, Braided bialgebras and quadratic bialgebras, Comm. Algebra 21 (1993), 17311749 .

[FRT] L. D. Faddeev, N. Yu. Reshetykhin and L. A. Takhtajan, Quantization of Lie groups and Lie algebras, Algebra and Analysis 1 (1987), 178-206.

[GM] X. Gomez and S. Majid, Braided Lie algebras and bicovariant differential calculi over co-quasitriangular Hopf algebras, math.QA/0112299. To appear in J. Algebra.

[HS] I. Heckenberger and K. Schmüdgen, Classification of bicovariant differential calculi on the quantum groups $S L_{q}(n+1)$ and $S p_{q}(2 n)$, J. Reine Angew. Math. 502 (1998), 141-162.

[KS] A. Klimyk and K. Schmüdgen, Quantum Groups and Their Representations, Texts and Monographs in Physics, Springer, 1997.

[LS] V. Lyubashenko and A. Sudbery, Generalized Lie algebras of type $A_{n}$, J. Math. Phys. 39 (1998), 3487-3504.

[Maj] S. Majid, Foundations of Quantum Group Theory, Cambridge Univ. Press, Cambridge, 1995.

[Maj-LN] S. Majid, Algebras and Hopf algebras in braided categories, in: Lecture Notes Pure Appl. Math. 158, Marcel Dekker, 1994, 55-105.

[Maj-93] S. Majid, Braided groups, J. Pure Appl. Algebra 86 (1993) 187-221.

[Maj-94] S. Majid, Quantum and braided Lie algebras, J. Geom. Phys. 13 (1994), 307-356.

[Maj-95] S. Majid, Solutions of the Yang-Baxter equations from braided Lie algebras and braided groups, J. Knot Theory Ramifications 4 (1995), 673-697.

[Maj-98] S. Majid, Classification of bicovariant differential calculi, J. Geom. Phys. 25 (1998), 119-140.

[Wor] S. L. Woronowicz, Differential calculus on compact matrix pseudogroups (quantum groups), Comm. Math. Phys. 122 (1989), 125-170. 